\title{
Correlations in a confined gas of harmonically interacting spin-polarized fermions
}

\author{
F. Brosens and J. T. Devreese* \\ Departement Natuurkunde, Universiteit Antwerpen (UIA), Universiteitsplein 1, B-2610 Antwerpen, Belgium \\ L. F. Lemmens \\ Departement Natuurkunde, Universiteit Antwerpen (RUCA), Groenenborgerlaan 171, B-2020 Antwerpen, Belgium
}

(Received 11 March 1998)

\begin{abstract}
For a fermion gas with equally spaced energy levels, the density and the pair correlation function are obtained. The derivation is based on the path integral approach for identical particles and the inversion of the generating functions for both static responses. The density and the pair correlation function are evaluated explicitly in the ground state of a confined fermion system with a number of particles ranging from 1 to 220 , and filling the Fermi level completely. [S1063-651X(98)12308-5]
\end{abstract}

PACS number(s): 05.30.-d, 03.75.Fi, 32.80.Pj

\section{INTRODUCTION}

The free energy and the static correlation functions of a gas of identical particles with equally spaced energy levels can be calculated exactly [1-3], using a combination of the path integral method [4], the method of symmetrized density matrices [5], and inversion techniques for generating functions $[3,6]$. The free energy, the internal energy, the specific heat, the moments of inertia [7], the density, and the pair correlation function have been worked out earlier for confined bosons. For the fermion case, the free energy, the ground state energy, and the energy of the Fermi level were also studied before [3]. Using the same formalism, in the present paper we consider the density and the pair correlation function of a spin-polarized fermion gas with equally spaced energy levels. Most of the calculations are analytical, and numerical work is only required for making the graphs. Nevertheless great attention had to be paid to the accurate calculability of the expressions in view of the sign problem originating from the statistics.

Inspired by recently observed Bose-Einstein condensation [8-10], much theoretical work has been done on boson models with equally spaced energy levels using other methods [11-17]. Analogous models for fermions, taking into account the confinement as well as the statistics, have been proposed and studied in Refs. [18,19] as a model for a quantum dot, and in Refs. [20,21] as a model for confined fermion alkali metal vapors.

The model that we used before, and that we also will investigate in this paper, consists of $N$ identical particles with mass $m$ in a harmonic one-body confinement potential given by

$$
V_{1}=\frac{m \Omega^{2}}{2} \sum_{j=1}^{N} \mathbf{r}_{j}^{2}
$$

and interacting with each other through a two-body potential given by:

\footnotetext{
*Also at Universiteit Antwerpen (RUCA), Groenenborgerlaan 171, B-2020 Antwerpen, and Technische Universiteit Eindhoven, NL 5600 MB Eindhoven, The Netherlands.
}

$$
V_{2}=-\frac{m \gamma}{4} \sum_{j, l=1}^{N}\left(\mathbf{r}_{j}-\mathbf{r}_{l}\right)^{2}
$$

This model has been studied for distinguishable particles [22], where it turns out to be equivalent to a set of oscillators. Three oscillators have a frequency $\Omega$ and are related to the degrees of freedom of the center of mass; the remaining internal degrees of freedom oscillate with a frequency $w=\sqrt{\Omega^{2}-N \gamma}$. The model has led to some controversy when put in an occupation number version to deal with the statistics of the particles [23]. Avoiding the occupation number representation by a direct projection on the symmetric or antisymmetric representations of the permutation group $S_{N}$, two things become clear: first the center-of-mass coordinate factorizes out, indicating the independence of the internal degrees of freedom; and second the propagator associated with the center of mass remains invariant under projection, indicating that the evolution of the center-of-mass degrees of freedom of distinguishable or indistinguishable oscillators are the same. This aspect of the model may be clarified by the introduction of the center-of-mass coordinate $R=(1 / N) \sum_{j=1}^{N} \mathbf{r}_{j}$ into the two-body potential

$$
V_{2}=-\frac{m N \gamma}{2} \sum_{l=1}^{N} \mathbf{r}_{l}^{2}+\frac{m N^{2} \gamma}{2} R^{2} .
$$

The rewritten two-body potential makes it clear that the center of mass oscillates with a frequency $\Omega=\sqrt{\left(N \gamma+w^{2}\right)}$ that is lower or higher than the frequency $w$ of the internal degrees of freedom depending on the sign of $\gamma$, which we earlier denoted by $\omega^{2}$ or $-\omega^{2}$ depending on the case. For repulsion $\gamma>0$, there is a stability constraint on the confinement potential: $\Omega$ has to be large enough to keep all the repelling particles together.

Because this condition on $\Omega$ depends on the number $N$ of particles, an approach with a fixed number of particles is mandatory from the very beginning, resulting in a constraint on the summation over the cycles in the cyclic decomposition of the permutations. We circumvented the complications of this constraint by first transforming to generating functions, and subsequently inverting the transforms to obtain the partition function, the density, and the pair correlation func- 
tion. The application of these mathematical techniques are among the main results of this paper. The fact that they allow one to derive explicit expressions for the density and the pair correlation function of an interacting fermion system is also an interesting result.

The paper is organized as follows. In Sec. II, we collect the expressions obtained before for the fermion case, and in Sec. III we present the calculation of the density and the pair correlation function in a general theoretical setting. We show how to evaluate these response functions for a system with a minimal internal energy and a given number of particles. In Sec. IV, we discuss the results, and put the model into perspective.

\section{FERMION OSCILLATORS}

In this section the basic formulas which have been derived before for identical particles (bosons or fermions) are summarized and rewritten in such a way that they are more appropriate for dealing with fermions, in particular in view of the numerical treatment.

\section{A. Recurrence relation approach}

In our path-integral treatment [1], a recurrence relation was obtained for the partition function $\mathbb{Z}_{I}(N)$ corresponding to the degrees of freedom with frequency $w$ in the relativecoordinate system. Introducing $b=e^{-\beta w}$ for brevity in the notations, we found that

$$
\mathbb{Z}_{I}(N)=\frac{1}{N} \sum_{m=0}^{N-1} \xi^{N-m-1}\left(\frac{b^{(1 / 2)(N-m)}}{1-b^{N-m}}\right)^{3} Z_{I}(m) .
$$

This recurrence relation applies for bosons $(\xi=+1)$ and for fermions $(\xi=-1)$. The subscript $I$ refers to identical particles, which can be specified to be fermions (subscript $F$ ) or bosons (subscript $B$ ). A similar recurrence technique was used in Ref. [2] to obtain the contribution of the relativecoordinate system to the spatial Fourier transform of the density

$$
\tilde{n}_{\mathbf{q}}=\frac{1}{N} \sum_{l=1}^{N} \frac{\xi^{l-1} \exp \left(-\frac{\hbar q^{2}}{4 m w} \operatorname{coth} \frac{1}{2} l \beta \hbar w\right)}{\left(2 \sinh \frac{1}{2} l \beta \hbar w\right)^{3}} \frac{Z_{I}(N-l)}{\mathbb{Z}_{I}(N)} .
$$

The center-of-mass contribution introduces the factor

$$
n_{\mathbf{q}}=\exp \left[-\frac{\hbar q^{2}}{4 m N}\left(\frac{\operatorname{coth} \frac{1}{2} \beta \hbar \Omega}{\Omega}-\frac{\operatorname{coth} \frac{1}{2} \beta \hbar w}{w}\right)\right] \tilde{n}_{\mathbf{q}} .
$$

For the Fourier transform of the pair correlation function, we found that

$$
\begin{aligned}
g_{\mathbf{q}}= & \frac{1}{N} \sum_{l=2}^{N} \frac{Z_{I}(N-l)}{Z_{I}(N)} \frac{\xi^{l-1} b^{(3 / 2) l} l-1}{\left(1-b^{l}\right)^{3}} \sum_{j=1}\left[\exp \left(-\frac{\hbar q^{2}}{2 m w} \frac{1}{Q_{l, j}(b)}\right)\right. \\
& \left.+\xi\left[Q_{l, j}(b)\right]^{3} \exp \left(-\frac{\hbar q^{2}}{2 m w} Q_{l, j}(b)\right)\right],
\end{aligned}
$$

where

$$
Q_{l, j}=\frac{1-b^{l}}{\left(1-b^{j}\right)\left(1-b^{l-j}\right)} .
$$

The center of mass does not contribute directly to the pair correlation function. It was shown in Ref. [3] that the actual numerical implementation for fermions of Eq. (2.1) suffers from a sign problem. Therefore, a generating function approach, followed by an inversion of the transform, turned out to be a more appropriate method of evaluation.

\section{B. Generating function approach}

The generating function $\Xi_{F}(\beta, u)$ for the partition function

$$
\Xi_{F}(\beta, u)=\sum_{N=0}^{\infty} Z_{F}(\beta \mid N) u^{N}
$$

was obtained before [3]. However, it should be noted that this function is a calculation tool to obtain the partition function $\mathbb{Z}_{F}(\beta \mid N)$, where the number $N$ of particles is given and not subject to fluctuations. A discussion of this point can be found in Ref. [24]. This discussion points out what the difference is between an ensemble-based approach to the problem (see, e.g., Ref. [25]) and quantum statistics for a finite number of particles. The generating function for the Fourier transform of the density is

$$
\mathcal{G}_{n}(u, \mathbf{q})=\sum_{N=0}^{\infty} Z_{F}(\beta \mid N) N \tilde{n}_{\mathbf{q}} u^{N} .
$$

The generating function for the Fourier transform of the pair correlation function is

$$
\mathcal{G}_{g}(u, \mathbf{q})=\sum_{N=0}^{\infty} \mathbb{Z}_{F}(\beta \mid N) N(N-1) g_{\mathbf{q}} u^{N} .
$$

The defining equations for the density and the pair correlation function

$$
\begin{aligned}
n(\mathbf{r})= & \frac{1}{N}\left\langle\sum_{l=1}^{N} \delta\left(\mathbf{r}-\mathbf{r}_{l}\right)\right\rangle=\int \frac{d \mathbf{q}}{(2 \pi)^{3}} n_{\mathbf{q}} e^{-i \mathbf{q} \cdot \mathbf{r}}, \\
g(\mathbf{r}) & =\frac{1}{N(N-1)}\left\langle\sum_{l=1, l^{\prime} \neq l}^{N} \delta\left(\mathbf{r}-\mathbf{r}_{l}+\mathbf{r}_{l^{\prime}}\right)\right\rangle \\
& =\int \frac{d \mathbf{q}}{(2 \pi)^{3}} g_{\mathbf{q}} e^{-i \mathbf{q} \cdot \mathbf{r}}
\end{aligned}
$$

are taken over from Ref. [2], and will be rewritten in a numerically tractable form. In comparison to Ref. [2], the nor- 
malization factor in the defining equation for the pair correlation function has been changed from $N$ to $N(N-1)$, such that $\int d^{3} \mathbf{r} g(\mathbf{r})=1$. Considering a model of $N$ fermions with parallel spin in a harmonic confinement potential, and with a quadratic interparticle interaction as discussed in Sec. I, the following generating functions are obtained:

$$
\begin{gathered}
\Xi_{F}(u)=\prod_{\nu=0}^{\infty}\left(1+u b^{3 / 2} b^{\nu}\right)^{(1 / 2)(\nu+1)(\nu+2)} \\
=\exp \left(\sum_{l=1}^{\infty} \frac{(-1)^{l-1}\left(b^{3 / 2} u\right)^{l}}{l\left(1-b^{l}\right)^{3}}\right), \\
\frac{\mathcal{G}_{n}(u, q)}{\Xi_{F}(u)}=\sum_{l=1}^{\infty} \frac{(-1)^{l-1}\left(u b^{3 / 2}\right)^{l}}{\left(1-b^{l}\right)^{3}} \exp \left(-\kappa^{2} \frac{1+b^{l}}{1-b^{l}}\right) \\
\frac{\mathcal{G}_{g}(u, \mathbf{q})}{\Xi_{F}(u)}=\sum_{l=2}^{\infty} \sum_{j=1}^{l-1} \frac{(-1)^{l-1}\left(u b^{3 / 2}\right)^{l}}{\left(1-b^{l}\right)^{3}} \\
\times\left[\exp \left(-\frac{2 \kappa^{2}}{Q_{l, j}}\right)-\left(Q_{l, j}\right)^{3} \exp \left(-2 \kappa^{2} Q_{l, j}\right)\right],
\end{gathered}
$$

with

$$
\kappa^{2}=\frac{\hbar q^{2}}{4 m w}
$$

This means that for a system of noninteracting oscillators with eigenfrequency $w, \Xi_{F}(u)$ is formally a "grandcanonical partition" function. However, strictly speaking, it is not the grand-canonical partition function of the system with interaction for two reasons: first, one has to take the center-of-mass correction into account; and second the eigenfrequency $w$ in the relative coordinate system depends on the number of particles. But, given $w$, the full mechanism of generating functions in the relative coordinate system is applicable, provided afterwards that the necessary center-ofmass corrections are taken into account.

\section{Density and pair correlation function}

From the Fourier transform of $\mathcal{G}_{n}(u, \mathbf{q})$ and $\mathcal{G}_{g}(u, \mathbf{q})$, the density in the relative coordinate system and the pair correlation function can be obtained in real space:

$$
\tilde{n}_{F}(\mathbf{r})=\left(\frac{m w}{\pi \hbar}\right)^{3 / 2} \frac{1}{N} \sum_{l=1}^{\infty} \frac{(-1)^{l+1}\left(u b^{3 / 2}\right)^{l}}{\left(1-b^{2 l}\right)^{3 / 2}} \exp \left(-\rho^{2} \frac{1-b^{l}}{1+b^{l}}\right) \text {, }
$$

$$
\begin{aligned}
g_{F}(\mathbf{r})= & \left(\frac{m w}{2 \pi \hbar}\right)^{3 / 2} \frac{1}{N} \sum_{l=2}^{\infty} \frac{\left(-u b^{3 / 2}\right)^{l}}{\left(1-b^{l}\right)^{3}} \\
& \times \sum_{j=1}^{l-1} Q_{l, j}^{3 / 2}\left(e^{-(1 / 2)\left(\rho^{2} / Q_{l, j}\right)}-e^{\left.-(1 / 2) \rho^{2} Q_{l, j}\right),}\right.
\end{aligned}
$$

where

$$
\rho^{2}=\frac{m w r^{2}}{\hbar}
$$

Including the center-of-mass correction, the Fourier transform of Eq. (2.2) has to be used instead of $\tilde{n}_{\mathbf{q}}$, giving

$$
n_{F}(\mathbf{r})=\left(\frac{m w}{\pi \hbar}\right)^{3 / 2} \frac{1}{N} \sum_{l=1}^{\infty} \frac{(-1)^{l-1}\left(u b^{3 / 2}\right)^{l}}{\left(1-b^{l}\right)^{3}} A_{l}^{3 / 2} e^{-\rho^{2} A_{l}},
$$

with

$$
A_{l}=\frac{1}{\frac{w}{\Omega} \frac{\operatorname{coth} \frac{1}{2} \beta \hbar \Omega}{N}-\frac{\operatorname{coth} \frac{1}{2} \beta \hbar w}{N}+\frac{1+b^{l}}{1-b^{l}}} .
$$

\section{Ground state correlations}

The expressions for the density and the pair correlation will be studied in the zero-temperature limit $b \rightarrow 0$, i.e., in the ground state. The relation between the number of particles and the fugacity implies:

$$
N=\sum_{\nu=0}^{\infty} \frac{1}{2}(\nu+1)(\nu+2) \frac{u b^{3 / 2} b^{\nu}}{1+u b^{3 / 2} b^{\nu}}=-\sum_{l=1}^{\infty} \frac{\left(-u b^{3 / 2}\right)^{l}}{\left(1-b^{l}\right)^{3}},
$$

in which case the chemical potential $\mu$ in the fugacity $u$ $=e^{\beta \mu}$ becomes the Fermi energy. Integrating the generating functions as follows:

$$
\begin{gathered}
\int_{0}^{u} \frac{\mathcal{G}_{n}\left(u^{\prime}, \mathbf{q}\right)}{u^{\prime}} d u^{\prime}=\sum_{N=0}^{\infty} \mathbb{Z}_{F}(N) n_{\mathbf{q}} u^{N}=\Xi_{F}(u)\left\langle n_{\mathbf{q}}\right\rangle, \\
\int_{0}^{u} \int_{0}^{u^{\prime}} \frac{\mathcal{G}_{g}\left(u^{\prime \prime}, \mathbf{q}\right)}{\left(u^{\prime \prime}\right)^{2}} d u^{\prime \prime} d u^{\prime}=\sum_{N=0}^{\infty} \mathbb{Z}_{F}(N) g_{\mathbf{q}} u^{N}=\Xi_{F}(u)\left\langle g_{\mathbf{q}}\right\rangle,
\end{gathered}
$$

the Fourier transform of the density and the pair correlation function become:

$$
\begin{gathered}
\left\langle n_{\mathbf{q}}\right\rangle=\frac{1}{\Xi_{F}(u)} \int_{0}^{u} \frac{1}{u^{\prime}} \Xi_{F}\left(u^{\prime}\right) \sum_{l=1}^{\infty} \frac{(-1)^{l-1}\left(u^{\prime} b^{3 / 2}\right)^{l}}{\left(1-b^{l}\right)^{3}} \exp \left(-\kappa^{2} \frac{1+b^{l}}{1-b^{l}}\right) d u^{\prime}, \\
\left\langle g_{\mathbf{q}}\right\rangle=\frac{1}{\Xi_{F}(u)} \int_{0}^{u} \int_{0}^{u^{\prime}} \frac{1}{\left(u^{\prime \prime}\right)^{2}} \Xi_{F}\left(u^{\prime \prime}\right) \sum_{l=2}^{\infty} \sum_{j=1}^{l-1} \frac{(-1)^{l-1}\left(u^{\prime \prime} b^{3 / 2}\right)^{l}}{\left(1-b^{l}\right)^{3}}\left[\exp \left(-\frac{2 \kappa^{2}}{Q_{l, j}}\right)-\left(Q_{l, j}\right)^{3} \exp \left(-2 \kappa^{2} Q_{l, j}\right)\right] d u^{\prime \prime} d u^{\prime}
\end{gathered}
$$


A straightforward but tedious calculation shows the mathematical equivalence between the expressions obtained in the recursion approach and those obtained by integrating the generating functions. For the density this calculation proceeds as follows:

$$
\begin{aligned}
\sum_{N=0}^{\infty} \mathbb{Z}_{F}(N) n_{\mathbf{q}} u^{N} & =\sum_{N=0}^{\infty} \mathbb{Z}_{F}(N) \sum_{k=N}^{\infty} \frac{u^{1+k}(-1)^{-N+k}}{(1+k)} \frac{\left(b^{-N+1+k}\right)^{3 / 2}}{\left(1-b^{-N+1+k}\right)^{3}} \exp \left(-\kappa^{2} \frac{1+b^{-N+1+k}}{1-b^{-N+1+k}}\right) \\
& =\sum_{k=0}^{\infty} \sum_{N=0}^{k} \mathbb{Z}_{F}(N) \frac{u^{1+k}(-1)^{-N+k}}{(1+k)} \frac{\left(b^{-N+1+k}\right)^{3 / 2}}{\left(1-b^{-N+1+k}\right)^{3}} \exp \left(-\kappa^{2} \frac{1+b^{-N+1+k}}{1-b^{-N+1+k}}\right) \\
& =\sum_{k=0}^{\infty} \sum_{N=0}^{k} \mathbb{Z}_{F}(N) \frac{u^{1+k}(-1)^{-N+k}}{(1+k)} \frac{\left(b^{-N+1+k}\right)^{3 / 2}}{\left(1-b^{-N+1+k}\right)^{3}} \exp \left(-\kappa^{2} \frac{1+b^{-N+1+k}}{1-b^{-N+1+k}}\right) \\
& =\sum_{k=0}^{\infty} u^{1+k} \sum_{l=0}^{k} \mathbb{Z}_{F}(k-l) \frac{(-1)^{l}\left(b^{l+1}\right)^{3 / 2}}{(1+k)\left(1-b^{l+1}\right)^{3}} \exp \left(-\kappa^{2} \frac{1+b^{l+1}}{1-b^{l+1}}\right) \\
& =\sum_{N=1}^{\infty} u^{N} \sum_{l=1}^{N} \mathbb{Z}_{F}(N-l) \frac{(-1)^{l-1}\left(b^{l}\right)^{3 / 2}}{N\left(1-b^{l}\right)^{3}} \exp \left(-\kappa^{2} \frac{1+b^{l}}{1-b^{l}}\right)
\end{aligned}
$$

or

$$
n_{\mathbf{q}}=\frac{1}{N} \sum_{l=1}^{N} \frac{\mathbb{Z}_{F}(N-l)}{\mathbb{Z}_{F}(N)} \frac{(-1)^{l-1}\left(b^{l}\right)^{3 / 2}}{\left(1-b^{l}\right)^{3}} \exp \left(-\kappa^{2} \frac{1+b^{l}}{1-b^{l}}\right) .
$$

The equivalence for the pair correlation function can be obtained along the same lines.

\section{DENSITY IN THE GROUND STATE}

In this section the ground state density will be calculated, thereby introducing the Fermi level explicitly. Simultaneously, the sum rules will be checked.

\section{A. Fourier transform of the density at low temperature}

Starting from the generating function (2.12), the series expansion of the exponent in $\mathcal{G}_{n}(u, q) e^{\kappa^{2}}$ as a Taylor series in $b$ gives

$$
\begin{aligned}
\frac{\mathcal{G}_{n}(u, q) e^{\kappa^{2}}}{\Xi_{F}(u)}= & \sum_{n=0}^{\infty} \frac{\left(-2 \kappa^{2}\right)^{n}}{n !} \\
& \times \sum_{k=0}^{\infty} \frac{\Gamma(k+3+n)}{\Gamma(k+1) \Gamma(3+n)} \frac{u b^{(3 / 2)+n+k}}{1+u b^{(3 / 2)+n+k}},
\end{aligned}
$$

for which the analytic continuation has to be found as a function of the fugacity $u=e^{\beta \mu}$ for $b=e^{-\beta \hbar w}$ arbitrarily small. If $L$ denotes the lowest unoccupied level, this expression becomes, using $u b^{3 / 2}=b^{-L+\alpha}$ (with $\alpha \rightarrow 0^{+}$):

$$
\begin{aligned}
\frac{\mathcal{G}_{n}(u, q) e^{\kappa^{2}}}{\Xi_{F}(u)}= & \sum_{n=0}^{\infty} \frac{\left(-2 \kappa^{2}\right)^{n}}{n !} \sum_{k=0}^{\infty} \frac{\Gamma(k+3+n)}{\Gamma(k+1) \Gamma(3+n)} \\
& \times \frac{b^{-L+\alpha+n+k}}{1+b^{-L+\alpha+n+k}} .
\end{aligned}
$$

In the low-temperature limit $(b \rightarrow 0)$ it is clear that $b^{-L+\alpha+n+k} /\left(1+b^{-L+\alpha+n+k}\right)$ tends to zero for $-L+n+k$ $\geqslant 0$, and to unity if $-L+n+k<0$. For $T \rightarrow 0$ the summations can then be restricted to $n \leqslant L-1$ and $k \leqslant L-1-n$, and $\mathcal{G}_{n}(u, q) e^{\kappa^{2}} / \Xi_{F}(u)$ becomes a polynomial in $\left(-2 \kappa^{2}\right)$ :

$$
\begin{aligned}
\frac{\mathcal{G}_{n}(u, q) e^{\kappa^{2}}}{\Xi_{F}(u)} & =\sum_{n=0}^{L-1} \frac{\left(-2 \kappa^{2}\right)^{n}}{n !} \sum_{k=0}^{L-1-n} \frac{\Gamma(k+n+3)}{\Gamma(k+1) \Gamma(n+3)} \\
& =\sum_{n=0}^{L-1} \frac{\Gamma(L+3)}{\Gamma(L-n) \Gamma(n+1) \Gamma(n+4)}\left(-2 \kappa^{2}\right)^{n} .
\end{aligned}
$$

We note in passing that this expression can also be written as $(1 / 6) L(L+1)(L+2){ }_{1} F_{1}\left(1-L ; 4 ; 2 \kappa^{2}\right)$, where ${ }_{1} F_{1}$ denotes the confluent hypergeometric function which is related to the generalized Laguerre polynomials $L_{n}{ }^{\alpha}(z)=[(1$ $+\alpha, n) / n !]_{1} F_{1}(-n ; 1+\alpha ; z)$, where $(a, n) \equiv a(a+1) \cdots(a$ $+n-1)=\prod_{k=0}^{n-1}(a+k)=[\Gamma(a+n) / \Gamma(a)]$.

For $q=0$, one readily finds $\mathcal{G}_{n}(u, 0) / \Xi_{F}(u)=N$. One then obtains from $\tilde{n}_{\mathbf{q}}=\mathcal{G}_{1}(u, q) / \mathcal{G}_{1}(u, q=0)$, in the limit $T \rightarrow 0$,

$$
\begin{aligned}
\tilde{n}_{\mathbf{q}}= & \exp \left(-\frac{1}{4} \frac{\hbar q^{2}}{m w}\right) \sum_{n=0}^{L-1} \frac{6 \Gamma(L)}{\Gamma(1+n) \Gamma(4+n) \Gamma(L-n)} \\
& \times\left(-\frac{\hbar q^{2}}{2 m w}\right)^{n}
\end{aligned}
$$

The Fourier transform of the density, including the centerof-mass correction, readily follows from Eq. (2.2). It essentially modifies the spatial decay, not the polynomial which arises from the fermion statistics. Taking into account that $\operatorname{coth} \frac{1}{2} \beta \hbar \Omega \rightarrow 1$ and coth $\frac{1}{2} \beta \hbar w \rightarrow 1$ for $\beta \rightarrow \infty$, it follows that

$$
\begin{aligned}
n_{\mathbf{q}}= & \exp \left(-\frac{1}{4} \frac{\hbar q^{2}}{m W}\right) \sum_{n=0}^{L-1} \frac{6 \Gamma(L)}{\Gamma(1+n) \Gamma(4+n) \Gamma(L-n)} \\
& \times\left(-\frac{\hbar q^{2}}{2 m w}\right)^{n},
\end{aligned}
$$


with

$$
W=w \frac{N}{N-1+\frac{w}{\Omega}} .
$$

\section{B. Ground state density}

The probability density of the fermions can be obtained from the Fourier transform $n_{F}(\mathbf{r})=\int\left[d \mathbf{q} /(2 \pi)^{3}\right] n_{F, \mathbf{q}} e^{-i \mathbf{q} \cdot \mathbf{r}}$. Using Eq. (3.5), the angular integrations are readily performed, and the remaining radial integrations are of the form

$$
\begin{aligned}
\int_{0}^{\infty} & \kappa^{1+2 n} e^{-\kappa^{2}} \sin (2 \kappa a) d \kappa \\
& =\frac{1}{2} e^{-a^{2}} \sum_{j=0}^{n}\left(\begin{array}{c}
1+2 n \\
2 j
\end{array}\right)(-1)^{n-j} a^{1+2 n-2 j} \Gamma\left(j+\frac{1}{2}\right) .
\end{aligned}
$$

The duplication rule $\sqrt{\pi} \Gamma(2 j+1)=4^{j} \Gamma\left(j+\frac{1}{2}\right) \Gamma(j+1)$ allows us to rewrite $\Gamma\left(j+\frac{1}{2}\right)$ in gamma functions with an integer argument. After some algebraic manipulations, one is left with

$$
n_{F}(\mathbf{r})=\left(\frac{m W}{\pi \hbar}\right)^{3 / 2} e^{-(W / w) \rho^{2}} \sum_{j=0}^{L-1} \frac{6 \Gamma(L) \Gamma(2+2 j)}{\Gamma(1+j) \Gamma(4+j) \Gamma(L-j)}\left(-\frac{2 W}{4 w}\right)^{j} \sum_{n=0}^{j} \frac{1}{\Gamma(j-n+1) \Gamma(2+2 n)}\left(-4 \frac{W}{w} \rho^{2}\right)^{n}
$$

The remaining double sum represents a polynomial in $\rho^{2}$, the numerical evaluation of which presents no difficulties. The probability density in the origin $r=0$ becomes

$$
n_{F}(\mathbf{0})=\left(\frac{m W}{\pi \hbar}\right)^{3 / 2 L-1} \sum_{j=0}\left(-\frac{1}{2} \frac{W}{w}\right)^{j} \frac{6 \Gamma(L) \Gamma(2+2 j)}{\Gamma^{2}(1+j) \Gamma(4+j) \Gamma(L-j)},
$$

in which the remaining sum can be identified to be the hypergeometric series ${ }_{2} F_{1}\left[\frac{3}{2}, 1-L ; 4 ; 2(W / w)\right]$, although this knowledge still requires further numerical treatment.

\section{Sum rule for the density}

One can check that the density satisfies the relation $\int d \mathbf{r} n_{F}(\mathbf{r})=1$, as it should. For that purpose, performing the angular integrations, one is left with

$$
\begin{aligned}
\int d \mathbf{r} n_{F}(\mathbf{r}) & =\frac{24}{\sqrt{\pi}} \sum_{j=0}^{L-1}\left(-\frac{1}{2} \frac{W}{w}\right)^{j} \frac{\Gamma(L) \Gamma(2+2 j)}{\Gamma(1+j) \Gamma(4+j) \Gamma(L-j)} \sum_{n=0}^{j} \frac{(-4)^{n}}{\Gamma(j+1-n) \Gamma(2+2 n)} \int_{0}^{\infty} e^{-\rho^{2} \rho^{2+2 n} d \rho} \\
& =6 \sum_{j=0}^{L-1}\left(-\frac{1}{2} \frac{W}{w}\right)^{j} \frac{\Gamma(L) \Gamma(2+2 j)}{\Gamma(1+j) \Gamma(4+j) \Gamma(L-j)} \sum_{n=0}^{j} \frac{(-1)^{n}}{\Gamma(-n+j+1) \Gamma(1+n)} .
\end{aligned}
$$

For $j=0$, the summation over $n$ yields 1 , whereas for $j>0$ the summation over $n$ gives the binomial series $(1-1)^{j}=0$. Therefore,

$$
\int d \mathbf{r} n_{F}(\mathbf{r})=\left.6\left(-\frac{1}{2} \frac{W}{w}\right)^{j} \frac{\Gamma(L) \Gamma(2+2 j)}{\Gamma(1+j) \Gamma(4+j) \Gamma(L-j)}\right|_{j=0}=1,
$$

and the required sum rule is indeed satisfied.

\section{Mean square distance}

An analogous calculation allows one to calculate the mean square distance to the origin, $\left\langle r^{2}\right\rangle_{F}=\int d \mathbf{r} r^{2} n_{F}(\mathbf{r})$, and to derive a scaling factor $\sqrt{\left\langle r^{2}\right\rangle_{F}}$ appropriate for distances. Similar to the procedure for the sum rule above, one finds

$$
\left\langle r^{2}\right\rangle_{F}=\frac{3 \hbar}{m W} \sum_{j=0}^{L-1}\left(-\frac{1}{2} \frac{W}{w}\right)^{j} \frac{\Gamma(L) \Gamma(2+2 j)}{\Gamma(1+j) \Gamma(4+j) \Gamma(L-j)} \sum_{n=0}^{j}(-1)^{n} \frac{3+2 n}{\Gamma(j+1-n) \Gamma(1+n)} .
$$

Separating out the contribution of $j=0$ to the summation, one is left with

$$
\left\langle r^{2}\right\rangle_{F}=\frac{3 \hbar}{m W}\left[\frac{1}{2}+\sum_{j=1}^{L-1}\left(-\frac{1}{2} \frac{W}{w}\right)^{j} \frac{\Gamma(L) \Gamma(2+2 j)}{\Gamma(1+j) \Gamma(4+j) \Gamma(L-j)} \sum_{n=0}^{j}(-1)^{n} \frac{3+2 n}{\Gamma(j+1-n) \Gamma(1+n)}\right] .
$$




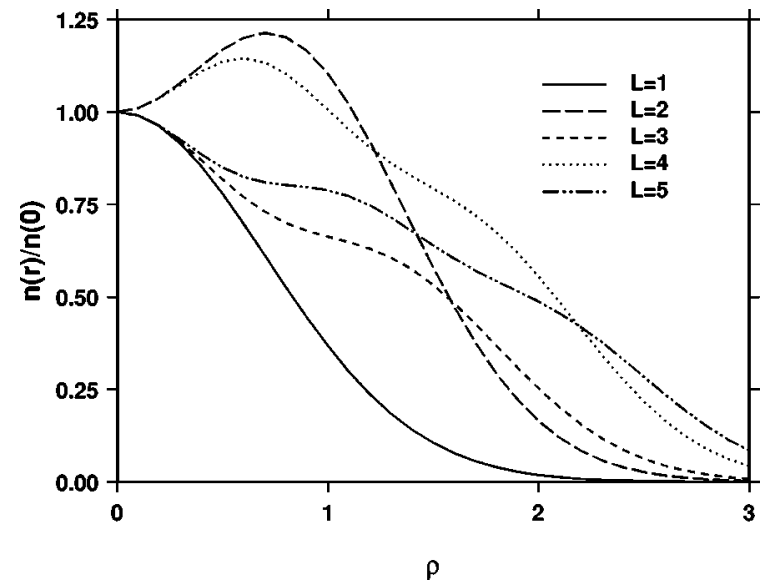

FIG. 1. Scaled density $n_{F}(\mathbf{r}) / n_{F}(\mathbf{0})$ as a function of $\rho=r \sqrt{m w / \hbar}$ for the lowest unoccupied levels characterized by $L=1,2,3,4$, and 5 .

Because $\quad \sum_{n=0}^{j}(-x)^{n}[(3+2 n) / \Gamma(n+1) \Gamma(-n+j+1)]$ $=[3 / \Gamma(1+j)](1-\varkappa)^{j}-[2 / \Gamma(j)](1-\varkappa)^{j-1} \varkappa$, the limit $\varkappa$ $\rightarrow 1$ can easily be taken:

$$
\begin{aligned}
\left\langle r^{2}\right\rangle_{F} & =\frac{3}{2} \frac{\hbar}{m W}+\frac{3}{4} \frac{\hbar}{m w}(L-1) \\
& =\frac{3}{2} \frac{\hbar}{m} \frac{w-W}{W w}+\frac{3}{4} \frac{\hbar}{m w}(L+1) .
\end{aligned}
$$

Hence the mean square distance of the spin-polarized fermions from the origin is proportional to $L \sim N^{1 / 3}$ for $N \gg 1$.

\section{Numerical results}

Having established the relevant distance scale, the scaled density $n_{F}(\mathbf{r}) / n_{F}(\mathbf{0})$ is plotted in Figs. 1 (for $L=1-5$ ) and 2 (for $L=6-10$ ) as a function of $\rho=r \sqrt{m w / \hbar}$ for the case of noninteracting fermions ( $W=\Omega=w$ ). The density in the origin for these cases is given in Table I. The density profiles can be compared with those obtained in Ref. [21], because the canonical ensemble and the quantum statistical partition function lead to the same predictions for this model provided

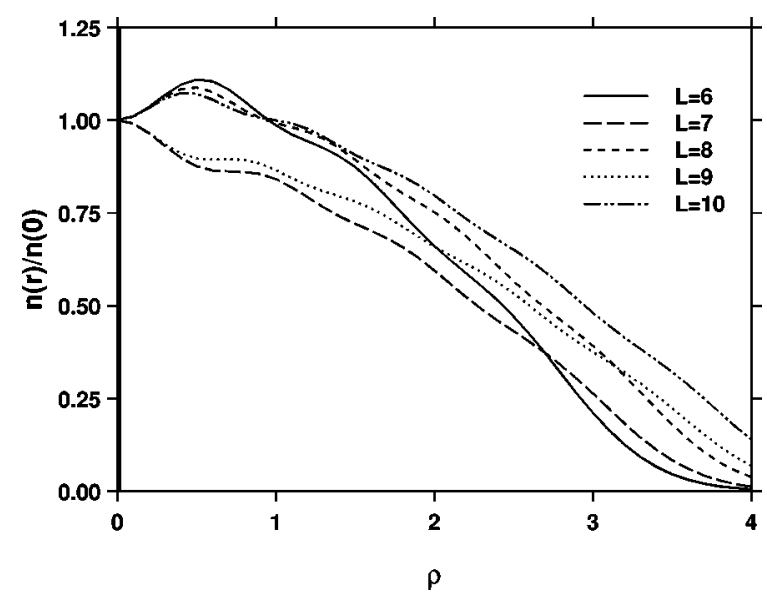

FIG. 2. Scaled density $n_{F}(\mathbf{r}) / n_{F}(\mathbf{0})$ as a function of $\rho=r \sqrt{m w / \hbar}$ for the lowest unoccupied levels characterized by $L=6,7,8,9$, and 10 .
TABLE I. Number of particles $N$ and density in the origin for the lowest unoccupied energy levels characterized by $L=1, \ldots, 10$.

\begin{tabular}{ccc}
\hline \hline$L$ & $N$ & $(\pi \hbar / m w)^{3 / 2} n_{F}(\mathbf{0})$ \\
\hline 1 & 1 & 1 \\
2 & 4 & $1 / 4$ \\
3 & 10 & $1 / 4$ \\
4 & 20 & $1 / 8$ \\
5 & 35 & $1 / 8$ \\
6 & 56 & $5 / 64$ \\
7 & 84 & $5 / 64$ \\
8 & 120 & $7 / 128$ \\
9 & 165 & $7 / 128$ \\
10 & 220 & $21 / 512$ \\
\hline \hline
\end{tabular}

the number of particles is given. Actually, we did not make a detailed comparison because our calculation is done for the ground state, while the calculations of Ref. [21] were done for finite temperature. Nevertheless the results for completely filled Fermi levels look very similar. The plots can also be made for the interacting case $\Omega \neq w$, but the differences are minor. For repulsive interactions the condition $0 \leqslant w \leqslant \Omega$ has to be taken into account. From the defining equation (3.6) for $W$, this leads to

$$
\frac{N}{N-1} \leqslant \frac{W}{w} \leqslant 1,
$$

and therefore the center-of-mass contribution to the density is washed out by the other degrees of freedom, except for a very limited number of fermions.

\section{PAIR CORRELATION FUNCTION IN THE GROUND STATE}

In this section we will repeat the same analysis as in Sec. III, but for the pair correlation function.

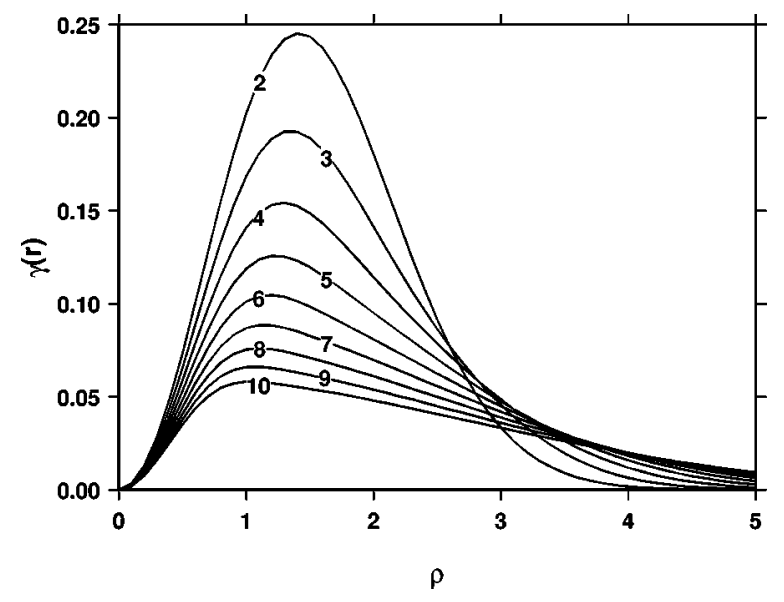

FIG. 3. Reduced pair correlation function $\gamma(\mathbf{r})$ $=g_{F}(\mathbf{r})(\pi \hbar / 2 m w)^{3 / 2}$ as a function of $\rho=r \sqrt{m w / \hbar}$ for the lowest unoccupied levels characterized by $L=2,3, \ldots, 10$, indicated by the numbers in the corresponding curves. 


\section{A. Ground state expressions}

In contrast to the calculation of the density, which was obtained from its Fourier transform, the calculation of the pair correlation function is easier in real space, starting from
Eq. (2.16). Because of the decay proportional to $e^{-(1 / 2) \rho^{2}}$, we expect that $g_{F}(\mathbf{r}) e^{(1 / 2) \rho^{2}}$ is a polynomial in $r$, which we want to be able to compute with sufficient accuracy in the lowtemperature limit $b \rightarrow 0$. From Eq. (2.16) one readily obtains

$$
\frac{g_{F}(\mathbf{r}) e^{(1 / 2) \rho^{2}}}{\left(\frac{m w}{2 \pi \hbar}\right)^{3 / 2}}=\frac{1}{N(N-1)} \sum_{l=1}^{\infty} \sum_{j=1}^{\infty} \frac{\left(-u b^{3 / 2}\right)^{j}}{\left(1-b^{j}\right)^{3 / 2}} \frac{\left(-u b^{3 / 2}\right)^{l}}{\left(1-b^{l}\right)^{3 / 2}} \frac{\exp \left[\frac{1}{2} \rho^{2}\left(1-\frac{1}{Q_{j+l, j}}\right)\right]-\exp \left[\frac{1}{2} \rho^{2}\left(1-Q_{j+l, j}\right)\right]}{\left(1-b^{j+l}\right)^{3 / 2}} .
$$

Expanding the exponential in a power series, and introducing the Taylor series for $Q_{j+l, j}$ [Eq. (2.5)] and for the denominators, the resulting 5-tuple series expansion can be rearranged in such a way that it can be summed in the limit $b \rightarrow 0$. The mathematical details of this derivation are given in Appendix A. The following expression was obtained for the lowtemperature limit:

$$
\begin{aligned}
g_{F}(\mathbf{r})= & \frac{e^{-(1 / 2) \rho^{2}}}{N(N-1)}\left(\frac{m w}{2 \pi \hbar}\right) \sum_{j=0}^{3 / 2} \sum_{k=0}^{j-1} \sum_{l=0}^{j} \frac{\left(\rho^{2} / 2\right)^{k+l}}{\Gamma(k+1) \Gamma(l+1) \Gamma(L-j) \Gamma(1+j-l) \Gamma(1+j-k)} \\
& \times\left(\frac{\Gamma^{2}\left(\frac{5}{2}+j-k-l\right) \Gamma\left(\frac{1}{2}+L-j+k+l\right)}{\Gamma\left(\frac{5}{2}-k\right) \Gamma\left(\frac{5}{2}-l\right) \Gamma\left(\frac{3}{2}+k+l\right)}-\frac{(-1)^{k+l} \Gamma^{2}\left(\frac{5}{2}+j\right) \Gamma\left(\frac{1}{2}+L-j\right)}{\Gamma\left(\frac{5}{2}+l\right) \Gamma\left(\frac{5}{2}+k\right) \Gamma\left(\frac{3}{2}\right)}\right) .
\end{aligned}
$$

The behavior in the origin is determined from $k=0$ and $l=0$, and obviously results in the expected result $g_{F}(\mathbf{0})=0$. In Fig. 3 , the pair correlation function of the spin-polarized fermions is shown as a function of $\rho$ for various Fermi levels.

\section{B. Sum rule for the pair correlation function}

An important check on the validity of the derivation above is provided by the condition that the pair correlation function has to satisfy the sum rule $\int d \mathbf{r} g_{F}(\mathbf{r})=1$. Performing the angular integrations, and using $\int_{0}^{\infty} \rho^{2}\left(\rho^{2} / 2\right)^{k+n} e^{-(1 / 2) \rho^{2}} d \rho=\sqrt{2} \Gamma\left(\frac{3}{2}\right.$ $+n+k)$, one obtains

$$
\begin{aligned}
\int d \mathbf{r} g_{F}(\mathbf{r})= & \frac{2}{N(N-1) \sqrt{\pi}} \sum_{j=0}^{L-1} \sum_{k=0}^{j} \sum_{l=0}^{j} \frac{\Gamma^{2}\left(\frac{5}{2}+j-k-l\right) \Gamma\left(\frac{1}{2}+L-j+k+l\right)}{\Gamma(k+1) \Gamma(l+1) \Gamma(L-j) \Gamma(1+j-l) \Gamma(-k+1+j) \Gamma\left(\frac{5}{2}-k\right) \Gamma\left(\frac{5}{2}-l\right)} \\
& -\frac{2}{N(N-1) \sqrt{\pi}} \sum_{j=0}^{L-1} \sum_{k=0}^{j} \sum_{l=0}^{j} \frac{(-1)^{l+k} \Gamma^{2}\left(\frac{5}{2}+j\right) \Gamma\left(\frac{3}{2}+l+k\right) \Gamma\left(\frac{1}{2}+L-j\right)}{\Gamma(k+1) \Gamma(l+1) \Gamma(L-j) \Gamma(1+j-l) \Gamma(-k+1+j) \Gamma\left(\frac{5}{2}+l\right) \Gamma\left(\frac{5}{2}+k\right)} .
\end{aligned}
$$

We checked analytically, for $L=2,3, \ldots, 10$, that these summations indeed yield 1 , and therefore are rather confident that it holds in general.

\section{Fourier transform}

Although it is possible to calculate the Fourier transform of $g_{F}(\mathbf{r})$, this calculation is rather involved, and it is easier to evaluate the zero-temperature limit of $g_{\mathbf{q}}$ directly from the generating function along the same lines as above. The actual calculation proceeds along the same lines as for the density, the Fourier transformation of the density, and the pair correlation function itself: expand the expressions in powers of $b$, and study the analytic continuation of $\mathcal{G}_{g}(u, \mathbf{q})$ in the fugacity $u=e^{\beta \mu}$ for $u b^{3 / 2}=b^{-L+\alpha}$. The calculation only differs in the details from the three examples in Sec. III A, III B, and IV A, and it seems pointless to report the full details of the calculation. Again, using $\kappa^{2}=\hbar q^{2} / 4 m w$, the result is 


$$
\begin{aligned}
\frac{\mathcal{G}_{g}(u, \mathbf{q}) e^{2 \kappa^{2}}}{\Xi_{F}(u)}= & \left(\sum_{n=0}^{L-1} \frac{\Gamma(L+3)}{\Gamma(1+n) \Gamma(L-n) \Gamma(4+n)}\left(-2 \kappa^{2}\right)^{n}\right)^{2}-\frac{1}{6} L(L+1)(L+2) \\
& -\sum_{j=1}^{L-1} \frac{1}{\Gamma(L-j)} \sum_{m=1}^{j} \frac{1}{m \Gamma(-m+1+j)} \sum_{n=j+1}^{m+j} \frac{\left(-2 \kappa^{2}\right)^{n}}{\Gamma^{2}(n-j)} \frac{\Gamma(2-j+n+L)}{(n-m) \Gamma(3+n) \Gamma(j-n+m+1)}
\end{aligned}
$$

One readily checks that this expression indeed has the correct long wavelength limit

$$
\frac{\mathcal{G}_{g}(u, \mathbf{0})}{\Xi_{F}(u)}=\left(\frac{1}{6} L(L+1)(L+2)\right)^{2}-\frac{1}{6} L(L+1)(L+2)=N(N-1) .
$$

With the normalization implied by the defining equation (2.10), one finds the following polynomial in $\kappa^{2}$ from $g_{\mathbf{q}}$ $=\mathcal{G}_{g}(u, \mathbf{q}) / \mathcal{G}_{g}(u, \mathbf{q}=\mathbf{0})$ :

$$
\begin{aligned}
N g_{\mathbf{q}} e^{2 \kappa^{2}=} & \left(\frac{1}{6} \frac{\Gamma(L+3)}{\Gamma(L)}+\sum_{n=1}^{L-1} \frac{\Gamma(L+3)}{\Gamma(1+n) \Gamma(L-n) \Gamma(4+n)}\left(-2 \kappa^{2}\right)^{n}\right)^{2}-\frac{1}{6} \frac{\Gamma(L+3)}{\Gamma(L)} \\
& -\sum_{j=1}^{L-1} \sum_{n=1}^{j} \frac{\left(-2 \kappa^{2}\right)^{n+j}}{\Gamma^{2}(n)} \frac{\Gamma(2+n+L)}{\Gamma(L-j) \Gamma(3+n+j)} \sum_{m=n}^{j} \frac{1}{(n+j-m) m \Gamma(j-m+1) \Gamma(m+1-n)} .
\end{aligned}
$$

Further simplifications are not easy to obtain, but this expression does not present numerical problems.

\section{CONCLUSION AND DISCUSSION}

In this paper we have shown that the density and the pair correlation function of the spin-polarized fermion oscillator model can be calculated taking exactly the statistics of the particles into account. We studied explicitly the ground state correlations of this confined fermion system for a set of particle numbers that correspond with fully occupied Fermi levels. One of the reasons for this limitation is that there is an additional simplification in the algebra, which is already tedious. Another reason is that this choice allows one to compare results calculated by inverting generating functions with results based on the iteration of the partition function. For a limited number of Fermi levels $(L=2,3,4)$ both methods have been used to check the results depicted in the figures.

The wiggles of the density as a function of the distance from the center are more pronounced, with an increasing number of particles, and are therefore clearly related to the increasing density of states at the Fermi level. Also, the trend that the most probable distance between a pair of fermions decreases while the range of distances wherever the pairs can be found increases with an increasing number of particles is worthwhile to point out. The mean distance and its variance are also calculated, because these quantities also provide important averages needed to perform a Jensen-Feynman variational calculation for a confined system of fermions with a realistic interparticle potential and/or a more complex confining potential. The model has also some importance in itself, because it can be used to test new approaches to Monte Carlo simulations of interacting fermions such as many-body diffusion [24,26,27].

In summary we have been able to calculate the thermodynamics and the static response functions of the harmonic model for a finite number of fermions without any recourse to the thermodynamical limit or to the theory of ensembles. Our approach is fully quantum mechanical, and relies on the probability assignment for quantum systems in equilibrium and as required by the statistics on the projection on the symmetric (bosons) or antisymmetric representation of the permutation group. Of course the generating function technique borrowed from the mathematics of stochastic processes together with path integral methods are essential in the formulation as well as in the evaluation of the thermodynamical quantities and the static response functions.

\section{ACKNOWLEDGMENTS}

Discussions with W. Krauth, F. Laloë, and Y. Kagan are acknowledged. Part of this work was performed in the framework of the FWO Project Nos. 1.5.729.94, 1.5.545.98, G.0287.95, G.0071.98, and WO.073.94N (Wetenschappelijke Onderzoeksgemeenschap over "Laagdimensionele systemen,' Scientific Research Community of the FWO on "Low Dimensional Systems"), the "Interuniversitaire Attractiepolen-Belgische Staat, Diensten van de Eerste Minister-Wetenschappelijke, Technische en Culturele aangelegenheden," and in the framework of the BOF NOI 1997 projects of the Universiteit Antwerpen. F. B. acknowledges the FWO for financial support.

\section{APPENDIX: MATHEMATICAL DETAILS OF THE PAIR CORRELATION FUNCTION}

In this appendix, a possible way to derive the lowtemperature limit (4.2) from Eq. (4.1) is given. We first expand the exponentials in Eqs. (4.1) in a power series, followed by filling out $Q_{j+l, j}$. This leads to 


$$
\begin{aligned}
\frac{g_{F}(\mathbf{r}) e^{(1 / 2) \rho^{2}}}{\left(\frac{m w}{2 \pi \hbar}\right)^{3 / 2}=} & \frac{1}{N} \sum_{n=0}^{\infty} \frac{1}{n !}\left(\frac{1}{2} \rho^{2}\right)^{n} \sum_{l=1}^{\infty} \sum_{j=1}^{\infty}\left(-u b^{3 / 2}\right)^{j+l}\left[b^{l}\left(1-b^{j}\right)+b^{j}\left(1-b^{l}\right)\right]^{n} \\
& \times\left(\frac{1}{\left(1-b^{j}\right)^{3 / 2}\left(1-b^{l}\right)^{3 / 2}\left(1-b^{j+l}\right)^{n+3 / 2}}-\frac{(-1)^{n}}{\left(1-b^{l}\right)^{n+3 / 2}\left(1-b^{j}\right)^{n+3 / 2}\left(1-b^{j+l}\right)^{3 / 2}}\right) .
\end{aligned}
$$

Using the binomial series $\left[b^{l}\left(1-b^{j}\right)+b^{j}\left(1-b^{l}\right)\right]^{n}=\sum_{k=0}^{n}\left(\begin{array}{l}n \\ k\end{array}\right) b^{l k} b^{j(n-k)}\left(1-b^{j}\right)^{k}\left(1-b^{l}\right)^{n-k}$, and the Taylor series expansion

$$
\frac{1}{\left(1-b^{j}\right)^{n+3 / 2}}=\sum_{l=0}^{\infty} \frac{b^{j l}}{l !} \frac{\Gamma\left(n+\frac{3}{2}+l\right)}{\Gamma\left(n+\frac{3}{2}\right)}
$$

for $b \rightarrow 0$, one obtains the following quite involved series expansion

$$
\begin{aligned}
\frac{g_{F}(\mathbf{r}) e^{(1 / 2) \rho^{2}}}{\left(\frac{m w}{2 \pi \hbar}\right)^{3 / 2}}= & \frac{1}{N} \sum_{n=0}^{\infty} \frac{1}{n !}\left(\frac{1}{2} \rho^{2}\right)^{n} \sum_{k=0}^{n}\left(\begin{array}{l}
n \\
k
\end{array}\right) \sum_{l=1}^{\infty} \sum_{j=1}^{\infty}\left(-u b^{3 / 2}\right)^{j+l} b^{l k} b^{j(n-k)} \sum_{m=0}^{\infty} \sum_{p=0}^{\infty} \sum_{q=0}^{\infty} \frac{b^{j m} b^{l p} b^{(j+l) q}}{m ! p ! q !} \\
& \times\left(\frac{\Gamma\left(\frac{3}{2}+m-k\right) \Gamma\left(\frac{3}{2}+p-n+k\right) \Gamma\left(\frac{3}{2}+q+n\right)}{\Gamma\left(\frac{3}{2}-k\right) \Gamma\left(\frac{3}{2}-n+k\right) \Gamma\left(\frac{3}{2}+n\right)}-\frac{\left.(-1)^{n} \Gamma\left(\frac{3}{2}+n-k+m\right) \Gamma\left(\frac{3}{2}+k+p\right) \Gamma\left(\frac{3}{2}+q\right)\right)}{\Gamma\left(\frac{3}{2}+n-k\right) \Gamma\left(\frac{3}{2}+k\right) \Gamma\left(\frac{3}{2}\right)} .\right.
\end{aligned}
$$

The summations over $l$ and $j$ can readily be performed, and using $u b^{3 / 2}=b^{-L+\alpha}$ the result can be written as

$$
\begin{aligned}
\frac{g_{F}(\mathbf{r}) e^{(1 / 2) \rho^{2}}}{\left(\frac{m w}{2 \pi \hbar}\right)^{3 / 2}}= & \frac{1}{N} \sum_{k=0}^{\infty} \sum_{n=0}^{\infty} \sum_{m=0}^{\infty} \sum_{p=0}^{\infty} \sum_{q=0}^{\infty} \frac{\left(\rho^{2} / 2\right)^{k+n}}{k ! n ! m ! p ! q !} \frac{b^{-L+\alpha+k+p+q}}{1+b^{-L+\alpha+k+p+q}} \frac{b^{-L+\alpha+n+m+q}}{1+b^{-L+\alpha+n+m+q}} \\
& \times\left(\frac{\Gamma\left(\frac{3}{2}-k+m\right) \Gamma\left(\frac{3}{2}-n+p\right) \Gamma\left(\frac{3}{2}+k+n+q\right)}{\Gamma\left(\frac{3}{2}-k\right) \Gamma\left(\frac{3}{2}-n\right) \Gamma\left(\frac{3}{2}+k+n\right)}-\frac{(-1)^{k+n} \Gamma\left(\frac{3}{2}+n+m\right) \Gamma\left(\frac{3}{2}+k+p\right) \Gamma\left(\frac{3}{2}+q\right)}{\Gamma\left(\frac{3}{2}+n\right) \Gamma\left(\frac{3}{2}+k\right) \Gamma\left(\frac{3}{2}\right)} .\right.
\end{aligned}
$$

The analysis of the zero-temperature limit $b \rightarrow 0$ then proceeds similarly to that for the density. The fraction $b^{-L+\alpha+k+p+q} /\left(1+b^{-L+\alpha+k+p+q}\right)$ tends to unity if $k+p+q \leqslant L-1$, and to zero otherwise. This means that the summations can be restricted to $k \leqslant L-1-q, p \leqslant L-1-q-k$, and $q \leqslant L-1$. A similar analysis applies to $b^{-L+\alpha+n+m+q /(1}$ $+b^{-L+\alpha+n+m+q}$ ), leaving a polynomial in $\rho^{2} / 2$ for $T \rightarrow 0$ :

$$
\begin{aligned}
\frac{g_{F}(\mathbf{r}) e^{(1 / 2) \rho^{2}}}{\left(\frac{m w}{2 \pi \hbar}\right)^{3 / 2}}= & \frac{1}{N} \sum_{q=0}^{L-1} \sum_{k=0}^{L-1-q} \sum_{n=0}^{L-1-q} \sum_{m=0}^{L-1-q-n} \sum_{p=0}^{L-1-q-k} \frac{\left(\rho^{2} / 2\right)^{k+n}}{k ! n ! m ! p ! q !} \\
& \times\left(\frac{\Gamma\left(\frac{3}{2}-k+m\right) \Gamma\left(\frac{3}{2}-n+p\right) \Gamma\left(\frac{3}{2}+k+n+q\right)}{\Gamma\left(\frac{3}{2}-k\right) \Gamma\left(\frac{3}{2}-n\right) \Gamma\left(\frac{3}{2}+k+n\right)}-(-1)^{k+n} \frac{\left.\Gamma\left(\frac{3}{2}+n+m\right) \Gamma\left(\frac{3}{2}+k+p\right) \Gamma\left(\frac{3}{2}+q\right)\right)}{\Gamma\left(\frac{3}{2}+n\right) \Gamma\left(\frac{3}{2}+k\right) \Gamma\left(\frac{3}{2}\right)} .\right.
\end{aligned}
$$

Also, the summations over $p$ and $m$ can be done analytically. These are of the form

$$
\sum_{p=0}^{M} \frac{\Gamma\left(k+\frac{3}{2}+p\right)}{p !}=\frac{2 k+3+2 M}{2 k+3} \frac{2^{-1-2 k-2 M} \sqrt{\pi} \Gamma(2 k+2 M+2)}{M !(k+M) !}=\frac{\Gamma\left(k+M+\frac{5}{2}\right)}{\left(k+\frac{3}{2}\right) M !}
$$


and hence, after some manipulations with the summation indices,

$$
\begin{aligned}
g_{F}(\mathbf{r})= & \frac{e^{-(1 / 2) \rho^{2}}}{N}\left(\frac{m w}{2 \pi \hbar}\right)^{3 / 2} \sum_{j=0}^{L-1} \sum_{k=0}^{j} \sum_{l=0}^{j} \frac{\left(\rho^{2} / 2\right)^{k+l}}{\Gamma(k+1) \Gamma(l+1) \Gamma(L-j) \Gamma(1+j-l) \Gamma(1+j-k)} \\
& \times\left(\frac{\Gamma^{2}\left(\frac{5}{2}+j-k-l\right) \Gamma\left(\frac{1}{2}+L-j+k+l\right)}{\Gamma\left(\frac{5}{2}-k\right) \Gamma\left(\frac{5}{2}-l\right) \Gamma\left(\frac{3}{2}+k+l\right)}-\frac{(-1)^{k+l} \Gamma^{2}\left(\frac{5}{2}+j\right) \Gamma\left(\frac{1}{2}+L-j\right)}{\Gamma\left(\frac{5}{2}+l\right) \Gamma\left(\frac{5}{2}+k\right) \Gamma\left(\frac{3}{2}\right)}\right) .
\end{aligned}
$$

[1] F. Brosens, J. T. Devreese, and L. F. Lemmens, Phys. Rev. E 55, 227 (1997).

[2] F. Brosens, J. T. Devreese, and L. F. Lemmens, Phys. Rev. E 55, 6795 (1997).

[3] F. Brosens, J. T. Devreese, and L. F. Lemmens, Phys. Rev. E 57, 3871 (1998).

[4] R. P. Feynman and A. R. Hibbs, Quantum Mechanics and Path Integrals (McGraw-Hill, New York, 1965).

[5] R. P. Feynman, Statistical Mechanics, a Set of Lectures (Benjamin, Reading, MA, 1972).

[6] L. F. Lemmens, F. Brosens, and J. T. Devreese (unpublished).

[7] F. Brosens, J. T. Devreese, and L. F. Lemmens, Phys. Rev. A 55, 2453 (1997).

[8] M. H. Anderson, J. R. Ensher, M. R. Matthews, C. E. Wieman, and E. A. Cornell, Science 269, 198 (1995).

[9] K. B. Davis, M. O. Mewes, M. R. Andrews, N. J. van Druten, D. S. Durfee, D. M. Kurn, and W. Ketterle, Phys. Rev. Lett. 75, 3969 (1995).

[10] C. C. Bradley, C. A. Sacket, J. J. Tollett, and R. G. Hulet, Phys. Rev. Lett. 75, 1687 (1995).

[11] S. Grossman and M. Holthaus, Z. Naturforsch. Teil A 50A, 323 (1995); 50A, 921 (1995).

[12] S. Grossman and M. Holthaus, Phys. Lett. A 208, 188 (1995).

[13] W. Ketterle and N. J. van Druten, Phys. Rev. A 54, 656 (1996).

[14] K. Kirsten and D. J. Toms, Phys. Rev. A 54, 4188 (1996).
[15] H. Haugerud, T. Haugset, and F. Ravndal, Phys. Rev. Lett. 225, 18 (1997).

[16] L. Cohen and C. Lee, J. Math. Phys. 26, 3105 (1985).

[17] W. Krauth, Phys. Rev. Lett. 77, 3695 (1996).

[18] N. F. Johnson and M. C. Payne, Phys. Rev. Lett. 67, 1157 (1991).

[19] W. Häusler, Z. Phys. B 99, 551 (1996).

[20] D. A. Butts and D. S. Rokhsar, Phys. Rev. A 55, 4346 (1997).

[21] J. Schneider and H. Wallis, Phys. Rev. A 57, 1253 (1998).

[22] G. W. Ford, M. Kac, and P. Mazur, J. Math. Phys. 6, 504 (1965); and represented in "Mathematical Physics in One Dimension,' edited by E. H. Lieb and D. C. Mattis (Academic, New York, 1966).

[23] D. J. Thouless, The Quantum Mechanics of Many-Body Systems, 2nd ed. (Academic, New York, 1972).

[24] L. F. Lemmens, F. Brosens, and J. T. Devreese, Phys. Rev. E 53, 4467 (1996).

[25] S. Grossmann and M. Holthaus, Phys. Lett. A 208, 188 (1995); Phys. Rev. E 54, 3495 (1996); H. D. Politzer, Phys. Rev. A 54, 5048 (1996); C. Herzog and M. Olshanii, ibid. 55, 3254 (1997).

[26] L. F. Lemmens, F. Brosens, and J. T. Devreese, Phys. Lett. A 189, 437 (1994).

[27] F. Brosens, J. T. Devreese, and L. F. Lemmens, Solid State Commun. 96, 137 (1995). 